\title{
Uncertainty in the Dynamics of Conservative Maps
}

\author{
Oliver Junge \\ Institute for Mathematics \\ University of Paderborn \\ Warburger Str. 100 \\ D-33098 Paderborn \\ Germany
}

\author{
Jerrold E. Marsden \\ Control and Dynamical Systems \\ California Institute of Technology, MC 107-81 \\ Pasadena, CA 91125 \\ USA
}

\author{
Igor Mezic \\ Department of Mechanical \\ and Environmental Engineering \\ UCSB \\ Santa Barbara, CA 93106 \\ USA
}

\begin{abstract}
This paper studies the effect of uncertainty, using random perturbations, on area preserving maps of $\mathbb{R}^{2}$ to itself. We focus on the standard map and a discrete Duffing oscillator as specific examples. We relate the level of uncertainty to the large scale features in the dynamics in a precise way. We also study the effect of such perturbations on bifurcations in such maps. The main tools used for these investigations are a study of the eigenfunction and eigenvalue structure of the associated Perron-Frobenius operator along with set oriented methods for the numerical computations.
\end{abstract}

\section{INTRODUCTION}

Uncertainty is obviously of central importance for dynamic and control systems both from a theoretical and a practical point of view. While this topic has received much attention in the control community, there is still much to learn about the effect of random perturbations on such systems. In this paper we focus our attention on perturbations of conservative systems as a first step towards studying mechanical systems (including molecular systems) in the presence of uncertainty. An important aspect of our approach is to try to extract the key dynamical features that survive in a noisy environment. One can make the case that such features are the most important ones to compute.

The specific context we work in is as follows. Let $f: X \rightarrow X$ be an area preserving map of a compact subset $X$ of $\mathbb{R}^{2}$ to itself. Let $\mathcal{B}$ be the Borel- $\sigma$-algebra and $m$ the standard volume measure on $X$. We model a perturbed version of the map $f$ by a stochastic process which maps a point $x \in X$ near the image point $f(x)$ with high probability. Formally, we consider a stochastic transition function $p: X \times \mathcal{B} \rightarrow[0,1]$ corresponding to a small random perturbation of $f$ in the sense of [1]. A typical small random perturbation of $f$ is given by letting $p_{\delta}(x, \cdot)$ be the uniform distribution on a $\delta$-ball around $f(x)$. This is in fact the type of perturbation we use in the computations.

Throughout the paper, we focus on two example systems,

1) the standard map

$$
\left(\begin{array}{l}
x_{1} \\
y_{1}
\end{array}\right)=\left(\begin{array}{l}
x+\varepsilon y+\varepsilon^{2} \rho \sin (2 \pi x) \\
y+\varepsilon \rho \sin (2 \pi x)
\end{array}\right)
$$

on the two-torus, as resulting from a symplectic time discretization of the ordinary differential equation for

Research partially supported by a Max Planck Research Award, NSFITR grant ACI-0204932 and AFOSR grant F49620-03-1-0096. the pendulum. We fix the step size $\varepsilon=1$ and view $\rho$ as a bifurcation parameter;

2) and the Duffing map,

$$
\left(\begin{array}{l}
x_{1} \\
y_{1}
\end{array}\right)=\left(\begin{array}{l}
x+\varepsilon y+\frac{\varepsilon^{2}}{2}\left(\rho x-x^{3}\right) \\
y+\frac{\varepsilon}{2}\left(\rho x-x^{3}+\rho x_{1}-x_{1}^{3}\right)
\end{array}\right)
$$

on $\mathbb{R}^{2}$, as resulting from applying the Newmark scheme to the Duffing equations (see, e.g., [10]). Here $\varepsilon$ is a fixed parameter (corresponding to the stepsize in the Newmark scheme) and $\rho \in \mathbb{R}$ is a bifurcation parameter.

\section{A. Stochastic transition functions}

A measure $\mu$ on $\mathcal{B}$ is called invariant for a stochastic transition function $p$, if

$$
\int p(x, A) \mu(d x)=\mu(A)
$$

for all $A \in \mathcal{B}$. A transition function $p$ is called reversible w.r.t an invariant probability measure $\mu$, if

$$
\int_{A} p(x, B) \mu(d x)=\int_{B} p(x, A) \mu(d x) .
$$

The value

$$
p(A, B):=\frac{1}{\mu(A)} \int_{A} p(x, B) \mu(d x)
$$

is the probability to map from set $A$ into set $B$ in one step. The corresponding probability for the time reversed system is given by

$$
\hat{p}(A, B)=\frac{\mu(B) p(B, A)}{\mu(A)} .
$$

A stochastic transition function with invariant measure $\mu$ is called uniformly ergodic, if there are constants $q<1$ and $M>0$, such that $\left\|p^{n}(x, \cdot)-\mu\right\| \leq M q^{n}$ for $n=0,1,2, \ldots$ and all $x \in X$.

An absolutely continuous transition function is of the form

$$
p(x, B)=\int_{B} k(f(x), y) d m(y),
$$

where $k: X \times X \rightarrow[0, \infty)$ is some suitable kernel. In this case, the transition function of the time reversed system is explicitly given by

$$
\hat{p}(x, A)=\int_{A} k(f(y), x) d m(y) .
$$




\section{B. Macroscopic dynamics}

It is instructive to first consider the situation when there is no noise. In this case, the Perron-Frobenius operator, acting on $L^{2}$, is given by $P h(x)=h \circ f^{-1}$ and its adjoint, the Koopman operator by $U h=h \circ f$. These are both examples of transfer operators ([9]). The spectral properties of these operators can be related to phase-space structure of the underlying map. In particular, invariant sets of $f$ can be obtained as level sets of functions in the eigenspace at 1 of $U$ (see e.g. [11]). In addition, invariant densities of $f$ are functions in the eigenspace at 1 of $P$. The operators $P$ and $U$ are unitary and thus their spectrum is confined to the unit circle in the complex plane. Their spectrum can be analyzed via the study of the simpler, symmetrized operator $(U+P) / 2$. Since $P$ is normal, any eigenfunction $h$ of $P$ at the eigenvalue $\lambda$ is an eigenfunction of $U$ at eigenvalue $\bar{\lambda}$, the complex conjugate of $\lambda$. In that case, we have

$$
\frac{1}{2}(U+P) h=\frac{(\lambda+\bar{\lambda})}{2} h=\operatorname{Re}(\lambda) h,
$$

and thus $h$ is an eigenvector of the symmetrized operator $(U+P) / 2$ associated with the eigenvalue $\operatorname{Re}(\lambda)$. In the case when $f$ is ergodic (and the eigenspace at 1 is onedimensional), all of the other eigenspaces are at most twodimensional. However, typical area-preserving maps show a more complicated structure in phase space where regular regions of periodic and quasi-periodic motion coexist with chaotic areas. An example of this is shown in Figure 1 for the standard map. In this case, the eigenspace at 1

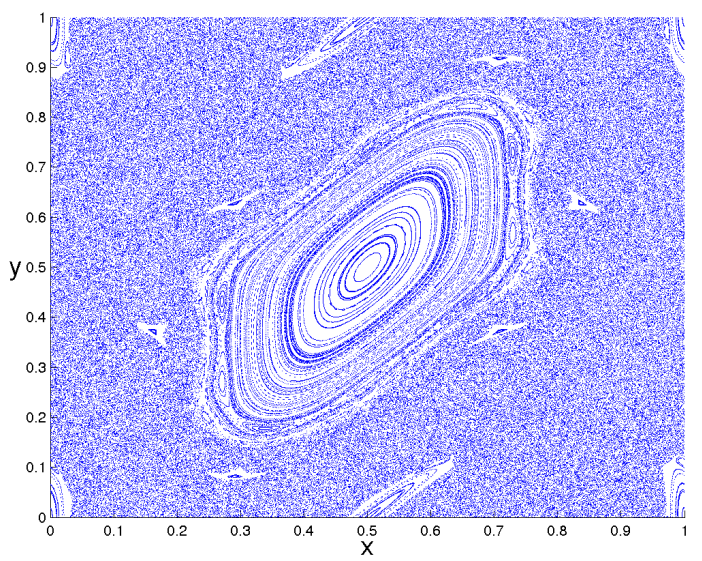

Fig. 1. Standard map: 500 iterates of 500 different initial conditions, chosen at random according to a uniform distribution, $\rho=0.3$.

can be obtained using the projection operator $P_{f} h=$ $(1 / n) \sum_{j=0}^{n-1} h \circ f^{j} h$. An example of such a computation is given in Figure 2.

In the presence of noise the decomposition of phase space into invariant sets with regular resp. chaotic dynamics is no longer possible. Due to the noise, the evolution may become transitive on the state space. However, for certain types of perturbations and if the perturbation is sufficiently small, certain invariant sets of the unperturbed map can

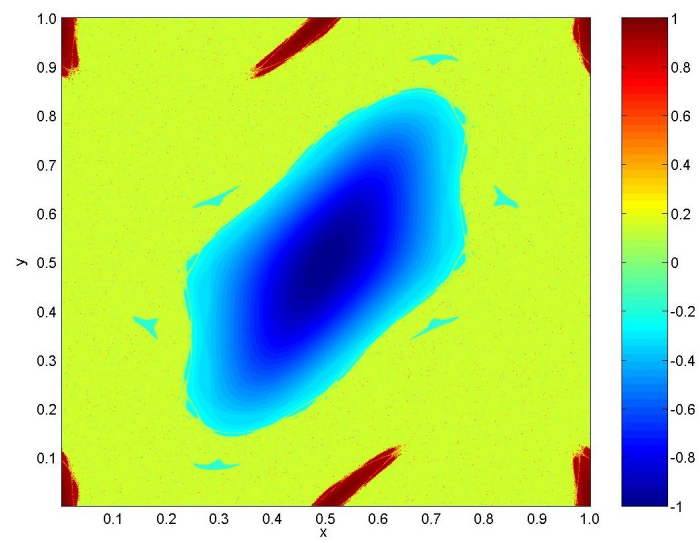

Fig. 2. Standard map: Invariant sets obtained from $P_{f} h$, for $h=$ $\cos (2 \pi y), \rho=0.3$.

still be recovered as almost invariant subsets. In fact, this is the more likely thing one will see in a more realistic noisy and uncertain model of a system. Intuitively, a set $A \subset X$ is almost invariant, if the invariance ratio of $A$, $p(A, A)$, is close to 1 , i.e. if the probability to stay within the set $A$ under the evolution is high. We refer to [2] for a more formal definition. For the perturbed system, it is therefore natural to ask for a decomposition of the state space into almost invariant sets when one is interested in a more macroscopic and robust description of the dynamical behavior. Thus, we aim at finding sets $A$ for which $p(A, A)$ is as large as possible. This question has been treated in [3], [4] in detail. It is a trivial (but important) observation, that

$$
p(A, A)=\hat{p}(A, A)=\frac{1}{2}(p(A, A)+\hat{p}(A, A)),
$$

i.e. when looking for sets that maximize $p(A, A)$, we might as well consider the transition function

$$
r(x, A)=\frac{1}{2}(p(x, A)+\hat{p}(x, A)),
$$

which is reversible.

\section{Transfer operators}

As mentioned before, spectral properties of transfer operators can be used to explore the macroscopic dynamics of the underlying system. For a given ("pointwise") evolution law, an associated transfer operator describes the evolution of probability measures or densities on the state space. Its spectrum can also be used to identify sets with a large almost invariance ratio; see e.g. [2].

For a deterministic map $f$, the Perron-Frobenius operator $P: \mathcal{M} \rightarrow \mathcal{M}$ is $P \mu(A)=\mu\left(f^{-1}(A)\right), A \in \mathcal{B}$, where $\mathcal{M}$ is the space of probability measures on $(X, \mathcal{B})$. The transfer operator of a stochastic transition function $p$ is given by

$$
P \mu(A)=\int p(x, A) d \mu(x), \quad \mu \in \mathcal{M} .
$$


In the case that $p$ is absolutely continuous, we can equivalently consider $P$ as an operator on $L^{2}(X)$, given by

$$
P h(y)=\int k(f(x), y) h(x) d m(x) \text {. }
$$

Note that for the standard inner product on $L^{2}$, the adjoint of $P$ is given by

$$
P^{T} g(x)=\int k(f(x), y) g(y) d m(y) .
$$

\section{A perturbed rotation on the circle}

As an example, we first study the spectral properties of the Perron-Frobenius operator for a perturbed rotation on the circle. In this example, spectral properties can be analytically computed and as a result are simple to understand. Let $f: S^{1} \rightarrow S^{1}, f(\theta)=\theta+\omega$, be a rotation on the circle, where $\omega$ is a constant. Note that this map preserves the Haar measure on the circle. The Perron-Frobenius operator associated with it is given by $P h(\theta)=h(\theta-\omega)$. The eigenfunctions of $P$ associated with the eigenvalues $\exp (-i 2 \pi n \omega), n \in \mathbb{N}$, are $\exp (i 2 \pi n \theta)$, which span $L^{2}\left(S^{1}\right)$. Consider the following stochastic perturbation of this conservative system: $f_{\delta}(\theta)=\theta+\omega+\xi$, where $\xi$ is a random variable uniformly distributed on the interval $[-\delta / 2, \delta / 2]$ for some $\delta>0$. The transfer operator associated with $f_{\delta}$ is

$$
P_{\delta} h(\theta)=\frac{1}{\delta} \int_{-\delta / 2}^{\delta / 2} h(\theta-\omega-\xi) d \xi .
$$

For $h(\theta)=\exp (i 2 \pi n \theta)$ we obtain

$$
\begin{aligned}
P_{\delta} h(\theta) & =\frac{1}{\delta} \int_{-\delta / 2}^{\delta / 2} \exp (i 2 \pi n(\theta-\omega-\xi)) d \xi \\
& =\frac{\sin (n \pi \delta)}{n \pi \delta} \exp (-i 2 \pi n \omega) \exp (i 2 \pi n \theta) .
\end{aligned}
$$

Thus $h_{n}(\theta)=\exp (i 2 \pi n \theta)$ are eigenfunctions of $P_{\delta}$ associated with the eigenvalues $\lambda_{n}=\frac{\sin (n \pi \delta)}{n \pi \delta} \exp (-i 2 \pi n \omega)$. For fixed $n$, if $\delta \rightarrow 0,\left|\lambda_{n}\right| \rightarrow 1$. In Figure 3 we show eigenvalues of $P_{\delta}$ for $\omega=\pi / 320$ and $\delta=0.01$. The eigenvalue with the largest modulus smaller than 1 is $\lambda_{1}=\frac{\sin (\pi \delta)}{\pi \delta} \exp (-i 2 \pi \omega)$, with the associated eigenfunction $\exp (i 2 \pi \theta)$. The adjoint operator of $P_{\xi}$ is the Koopman operator $U_{\delta} h(\theta)=\frac{1}{\delta} \int_{-\delta / 2}^{\delta / 2} h(\theta+\omega+\xi) d \xi$. In this case the Perron-Frobenius and Koopman operators are normal and thus the symmetrized operator $\left(P_{\delta}+U_{\delta}\right) / 2$ has eigenvalues $(\sin (n \pi \delta)(n \pi \delta) \cos (2 \pi n \omega))$ and the same eigenfunctions as $P_{\delta}$. For small $\omega$ the second largest eigenvalue is obtained for $n=1$. The associated eigenfunctions are given by $c_{1} \sin (2 \pi \theta)+c_{2} \cos (2 \pi \theta)$. Clearly any two arcs that split the circle in two parts are almost invariant sets.

\section{E. Eigenvalues and almost invariance}

For a reversible and uniformly ergodic absolutely continuous stochastic transition function $p$, the essential spectral radius of the corresponding transfer operator $P: L^{2} \rightarrow L^{2}$ is bounded away from one, i.e. $\sigma(P) \subset[\ell, r] \cup\{1\}$ with

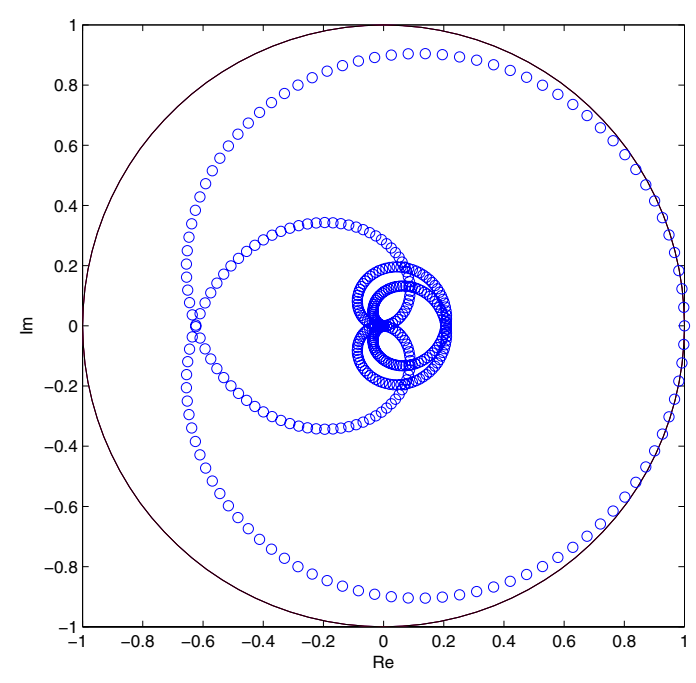

Fig. 3. Eigenvalues of $P_{\delta}$ for $\omega=\pi / 320$ and $\delta=0.01$.

$r<1$; see [6]. By combining Theorem 3.1 and Corollary 4.33 of [6], we obtain:

Proposition 1 Let the absolutely continuous stochastic transition function $p$ be reversible and uniformly ergodic. Assume that

$$
\sigma(P) \subset[\ell, r] \cup\left\{\lambda_{2}, 1\right\}
$$

with $r<\lambda_{2}$ and $\lambda_{2}$ a simple eigenvalue of $P$. Then for any set $A \in \mathcal{B}$

$$
1-\lambda_{2} \leq p\left(A, A^{C}\right)+p\left(A^{C}, A\right) \leq 1-\kappa \lambda_{2}
$$

where $0<\kappa \leq 1$.

In fact, one has (see [6])

$$
\kappa=\sqrt{\frac{\mu\left(A^{C}\right)}{\mu(A)}} \int_{A} v_{2} d \mu-\sqrt{\frac{\mu(A)}{\mu\left(A^{C}\right)}} \int_{A^{C}} v_{2} d \mu
$$

where $v_{2}$ is the eigenvector at $\lambda_{2}$. Note that $\kappa$ will be maximal if the set $A$ is the set on which $v_{2}$ is positive. In fact, the strategy of using the sign structure of the eigenvectors associated to eigenvalues $\neq 1$ in order to detect almost invariant sets has been proposed in [2] and already successfully been applied in different situations, in particular in order to identify conformations of molecules, see [5], [12].

In Figure 4 we show six eigenvectors of the reversibilized transfer operator of the unperturbed standard map. Clearly the almost invariant sets defined by the positive resp. negative components of these modes are in very good agreement with invariant sets of the unperturbed system (c.f. Figure 1) for the eigenvectors $v_{2}, \ldots, v_{6}$. The eigenvector $v_{7}$ decomposes the "chaotic sea" itself. 


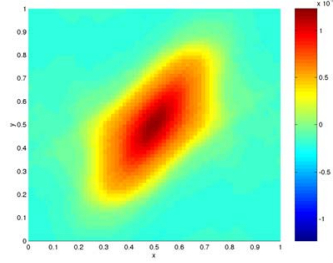

(a) $v_{2}$

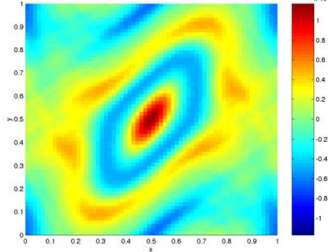

(c) $v_{4}$

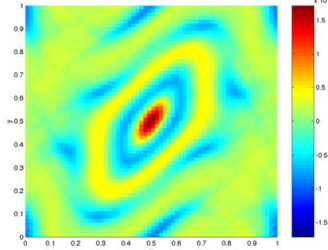

(e) $v_{6}$

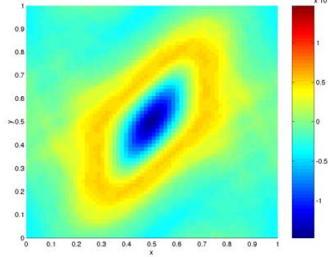

(b) $v_{3}$

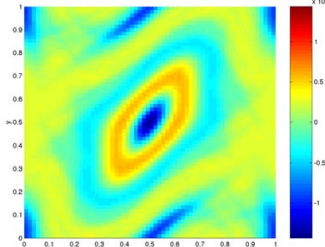

(d) $v_{5}$

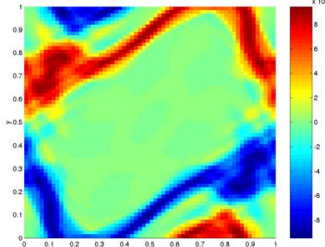

(f) $v_{7}$
Fig. 4. Standard map: eigenvectors $v_{2}, \ldots, v_{7}$ to the six largest eigenvalues (except 1) $\lambda_{2}<\cdots<\lambda_{7}$ of the reversibilized transfer operator of the unperturbed system on a partition of $2^{12}$ boxes, $\rho=0.3$.

\section{F. Discretization}

Numerically, we need to work with a finite dimensional subspace $L_{d}$ of $L^{2}$ and a corresponding approximation of $P_{\delta}$ on $L_{d}$. A common ansatz is to let $L_{d}$ be the subspace of step-functions that are piecewise constant on the elements of a partition of $X$. More precisely, let $\mathcal{P}=\left\{B_{1}, \ldots, B_{d}\right\} \subset$ $\mathcal{B}$ such that $\mu\left(B_{i} \cap B_{j}\right)=0$ for $B_{i}, B_{j} \in \mathcal{P}, B_{i} \neq B_{j}$ and $\bigcup_{i=1}^{d} B_{i}=X$. Let $L_{d}=\operatorname{span}\left\{\chi_{B_{1}}, \ldots, \chi_{B_{d}}\right\}$ and let $Q_{d}: L^{2} \rightarrow L_{d}$ be the orthogonal projection onto $L_{d}$. The discretized transfer operator $P_{d}$ is defined as $P_{d}=Q_{d} P_{\delta}$. It is easy to see (see e.g. [2]) that in this case the discretized operator can be represented by a stochastic matrix with entries

$$
p_{i j}=\frac{m\left(f^{-1}\left(B_{i}\right) \cap B_{j}\right)}{m\left(B_{j}\right)}, \quad i, j=1, \ldots, d .
$$

For bounded small perturbations of the map $f$, i.e. if $p_{\delta}(x, \cdot)$ has sufficiently small support, this transition matrix is sparse and even if the number $d$ of partition elements is quite large, a couple of eigenvalues and corresponding eigenvectors can efficiently be computed by Arnoldi methods as e.g. implemented in ARPACK [8] (which is available as the eigs command in MATLAB).

In Figure 5 we show the eigenvector to the second largest eigenvalue of the reversibilized discretized transfer operator $R_{d}=\left(P_{d}+P_{d}^{T}\right) / 2$ for the standard map, computed on four different partitions of the phase space. Note that the overall shape of the associated almost invariant decomposition is already quite well resolved on a partition with only 256 boxes.

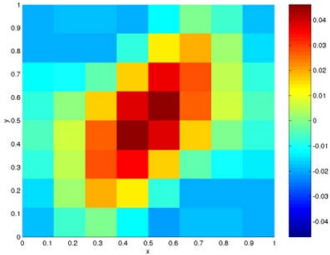

(a) $2^{6}$ boxes

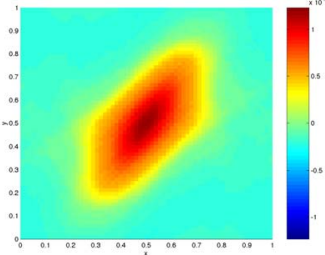

(c) $2^{12}$ boxes

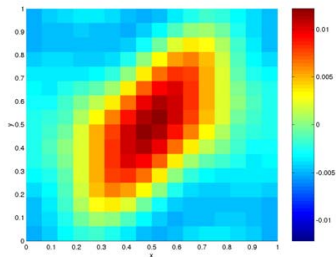

(b) $2^{8}$ boxes

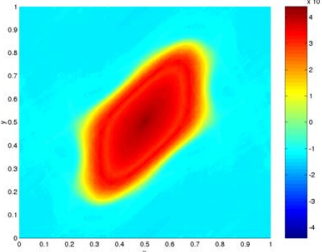

(d) $2^{16}$ boxes
Fig. 5. Standard map: eigenvector to the second largest eigenvalue of the reversibilized discretized transfer operator of the unperturbed system for different partitions, $\rho=0.3$.

\section{Spectral Properties of the Transfer OPERATOR}

A. The dependence of the spectrum on the perturbation size

Let us focus on a particular perturbation of the given map $f$ : We consider

$$
k_{\delta}(f(x), y)=\frac{1}{\mu\left(B_{\delta}(0)\right)} \chi_{B_{\delta}(f(x))}(y) .
$$

In [7] we prove:

Proposition 2 If $A$ is an invariant set of the unperturbed system, then the probability $p_{\delta}\left(A, A^{C}\right)$ to map from $A$ into its complement $A^{C}$ under the perturbed system can be estimated as

$$
p_{\delta}\left(A, A^{C}\right) \leq \frac{\text { length }(\partial A)}{\mu(A)} \delta+\mathcal{O}\left(\delta^{2}\right)
$$

as $\delta \rightarrow 0$.

Combining this estimate with Proposition 1, we obtain 
Theorem 1 Let $A$ be an invariant set of the unperturbed map $f$. Assume that the reversibilized small random perturbation $r_{\delta}=\frac{1}{2}\left(p_{\delta}+\hat{p}_{\delta}\right)$ of $f$ is uniformly ergodic. Then

$$
1-\lambda_{2} \leq \frac{\text { length }(\partial A)}{\mu(A) \mu\left(A^{C}\right)} \delta+\mathcal{O}\left(\delta^{2}\right)
$$

Proof: Note that $\mu(A)+\mu\left(A^{C}\right)=1$ and length $(\partial A)=$ length $\left(\partial A^{C}\right)$.

Note that the constant on the right hand side relates the length of the boundary of $A$ to its volume. In particular this means that a larger eigenvalue corresponds to an almost invariant set of larger volume. So in this sense larger eigenvalues detect "more important" almost invariant sets.

In Figure II-A we show how the four largest real eigenvalues of the discretized transfer operator for the standard map depend on the perturbation size $\delta$. The computation of the transition matrix is based on a partition of $2^{14}$ boxes. We used 16 test points on a regular grid in each box of the covering and 256 points on a regular grid in each $\delta$ neighborhood of the corresponding image points in order to sample $p_{\delta}(x, \cdot)$. Note that this numerical result is in very good agreement with Theorem 1.

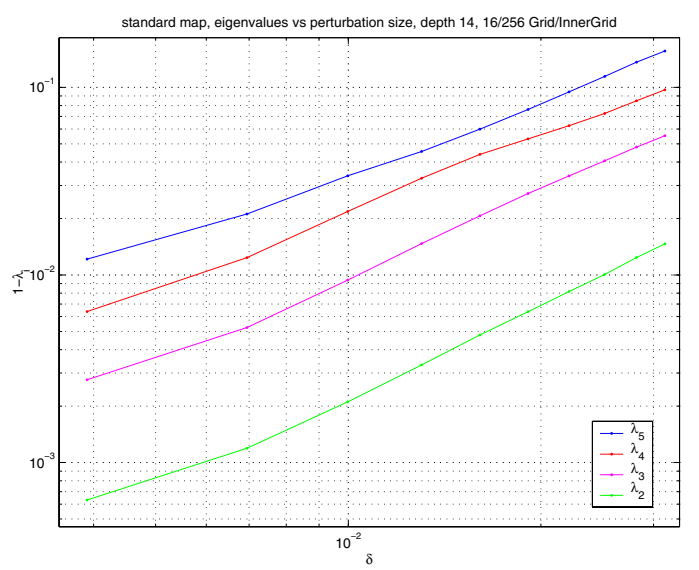

Fig. 6. Standard map: the four largest real eigenvalues (except 1) in dependence of the perturbation size.

\section{B. Spectrum for a fixed value of the bifurcation parameter}

Suppose that we are given a symmetry transformation $\kappa: X \rightarrow X$, such that $\kappa^{2}=i d$ and the map $f$ satisfies the equivariance condition

$$
\kappa \circ f=f^{-1} \circ \kappa .
$$

Define the action of $\kappa: L^{2} \rightarrow L^{2}$ by $\kappa h=h \circ \kappa$.

Proposition 3 Suppose that the kernel $k_{\delta}$ satisfies

$$
k_{\delta}(f(x), y)=k_{\delta}\left(\kappa \circ f^{-1}(y), \kappa(x)\right)
$$

then

$$
\kappa P_{\delta}=P_{\delta}^{T} \kappa
$$

Proof: We compute

$$
\begin{aligned}
P_{\delta}^{T} \kappa h(x) & =\int k_{\delta}(f(x), y) h \circ \kappa(y) d \mu(y) \\
& =\int k_{\delta}\left(\kappa \circ f^{-1}(y), \kappa(x)\right) h \circ \kappa(y) d \mu(y) \\
& =\int k_{\delta}\left(\kappa\left(y^{\prime}\right), \kappa(x)\right) h \circ \kappa \circ f\left(y^{\prime}\right) d \mu\left(y^{\prime}\right),
\end{aligned}
$$

where the latter equality follows using the change of variables $y^{\prime}=f^{-1}(y)$ and the fact that $f$ is area preserving. Using the equivariance condition (17) we continue

$$
\begin{aligned}
& =\int k_{\delta}\left(\kappa\left(y^{\prime}\right), \kappa(x)\right) h \circ f^{-1} \circ \kappa\left(y^{\prime}\right) d \mu\left(y^{\prime}\right) \\
& =\int k_{\delta}(\tilde{y}, \kappa(x)) h \circ f^{-1}(\tilde{y}) d \mu(\tilde{y}) \\
& =\int k_{\delta}(f(\hat{y}), \kappa(x)) h(\hat{y}) d \mu(\hat{y}) \\
& =P_{\delta} h \circ \kappa(x)=\kappa P_{\delta} h(x),
\end{aligned}
$$

where, again, we performed two changes of variables, exploiting the area preservation property of $\kappa$.

Corollary 1 Let $h \in L^{2}$ be an eigenfunction of $P_{\delta}$ at the eigenvalue $\lambda$, then $\kappa h$ is an eigenfunction of $P_{\delta}^{T}$ at the eigenvalue $\lambda$.

Numerical support for this Corollary is shown in Figure 7. We consider the Duffing map, for which a suitable symmetry is given by $\kappa(x, y)=(-x, y)$. For $\varepsilon=0.1$ and $\rho=-0.2$, we computed the transition matrix on a covering of $2^{12}$ boxes, using 100 test points on a regular grid. We set $\delta=0$, i.e. did not use an explict perturbation-still one may view the discretization as inducing one. The figure shows $e^{i \pi} \kappa h$ in the left column, where $h$ is the eigenvector of $P_{\delta}$ and the eigenvector of $P_{\delta}^{T}$ in the right column, both for the same eigenvalue $\lambda=0.9902+i 0.0647$.
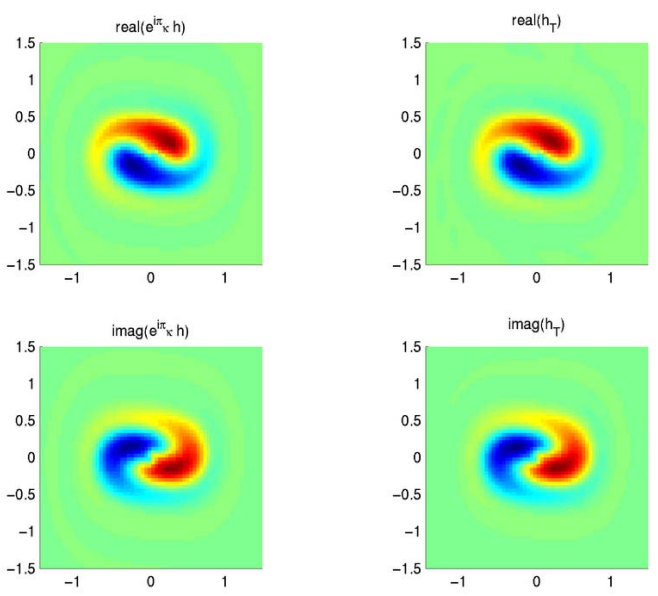

Fig. 7. Duffing map: Comparison of $e^{i \pi} \kappa h$ (left column), where $h$ is the eigenvector of $P_{\delta}$, and the corresponding eigenvector of $P_{\delta}^{T}$ (right column). 


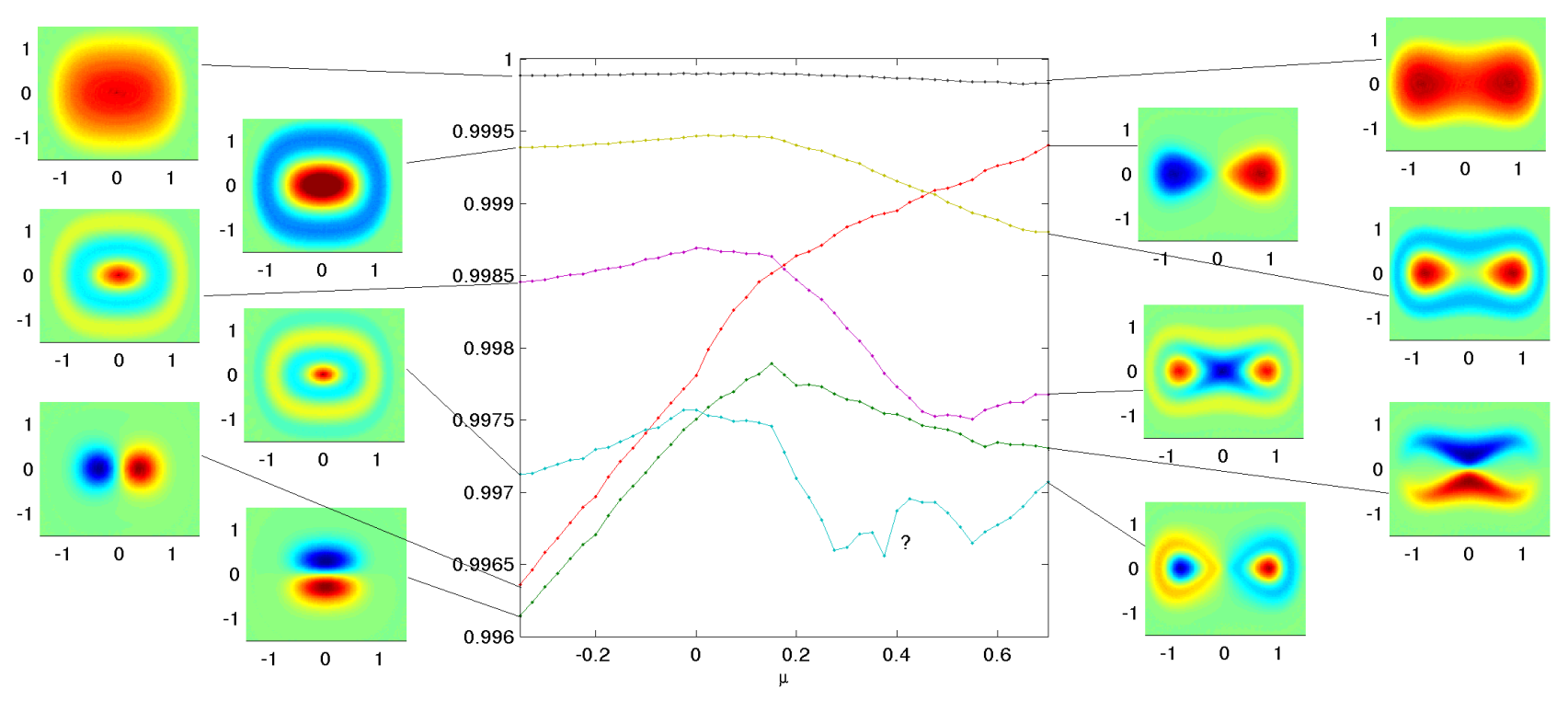

Fig. 8. Duffing: The six largest eigenvalues of the reversibilized discretized transfer operator in dependence of the bifurcation parameter $\rho$, together with the corresponding eigenmodes $(\varepsilon=0.1$, depth $=14, \delta=0)$.

\section{Spectrum in dependence of the bifurcation parameter}

As a bifurcation parameter is varied, it is natural to expect the spectrum of the transfer operator to change. In particular, we expect the corresponding eigenmodes to reflect changes in the global dynamical behavior of the system. As an example situation we consider the dynamics of the Duffing map as the parameter $\rho$ is varied. It is well known that at $\rho=0$ the origin undergoes a pitchfork bifurcation, leading to two stable equilibria for $\rho>0$. In Figure 8 we show how part of the spectrum of the reversibilized transfer operator depends on $\rho \in[-0.35,0.7]$. One particular eigenfunction (associated to the eigenvalue represented by the red curve in Figure 8) appears to become the dominant one as $\rho$ changes from -0.35 to 0.7 . For positive $\rho$, the positive resp. negative components of this eigenfunction are associated to neighborhoods of the two equilibria which are created in the pitchfork bifurcation, bounded by the homoclinic orbits of the origin. For negative $\rho$ however, this geometric situation is not present, but still the sign structure of this eigenmode defines a qualitatively similar decomposition of the state space into two almost invariant sets. Recall that we are considering the reversibilized operator here (as justified by (8)), so these modes should not directly be compared to the ones shown in Figure 7.

\section{CONCLusions.}

This paper demonstrates that the framework of transfer operators provides an efficient way to study the effect of uncertainty on area preserving maps. In particular, we show how the level of uncertainty affects the macroscopic dynamical behaviour in a quantitative way. Using the Duffing oscillator as example, we also explore how uncertainty may affect a bifurcation.

\section{REFERENCES}

[1] Y. Kifer, Random Perturbations of Dynamical Systems. Birkhäuser, 1988.

[2] M. Dellnitz and O. Junge, "On the approximation of complicated dynamical behavior," SIAM J. Numer. Anal., vol. 36, no. 2, pp. 491$515,1999$.

[3] G. Froyland and M. Dellnitz, "Detecting and Locating Near-Optimal Almost-Invariant Sets and Cycles", SIAM Journal on Scientific Computing, vol. 24, no. 6, pp. 1839-1863, 2003.

[4] G. Froyland, " $\mu$ Almost-invariant sets and Adaptive Boundary Refinement", Preprint.

[5] P. Deuflhard and M. Dellnitz and O. Junge and Ch. Schütte, "Computation of Essential Molecular Dynamics by Subdivision Techniques I: Basic Concept", in P. Deuflhard,J. Hermans, B. Leimkuhler and A.E. Mark, S. Reich and R.D. Skeel (eds.) "Computational Molecular Dynamics: Challenges, Methods, Ideas", Lecture Notes in Computational Science and Engineering, vol. 4, pp. 98-115, Springer, 1998.

[6] W. Huisinga, "Metastability of markovian systems: A transfer operator approach in application to molecular dynamics," Ph.D. dissertation, Free University Berlin, 2001.

[7] O. Junge, J. E. Marsden and I. Mezic, "Random Perturbations of Conservative Maps," in preparation.

[8] R. B. Lehoucq and D. C. Sorensen, "Deflation techniques for an implicitly restarted Arnoldi iteration", SIAM J. Matrix Analysis and Applications, vol. 17, no. 4, pp. 789-821, 1996.

[9] A. Lasota and M. C. Mackey, Chaos, Fractals and Noise. SpringerVerlag, 1994.

[10] J. E. Marsden and M. West, "Discrete mechanics and variational integrators", Acta Numerica vol. 10, pp. 357-514, 2001.

[11] I. Mezić and A. Banaszuk "Comparison of complex systems", Physica D (submitted), 2004.

[12] Ch. Schütte and W. Huisinga and P. Deuflhard, "Transfer Operator Approach to Conformational Dynamics in Biomolecular Systems", in B. Fiedler (ed.): Ergodic Theory, Analysis, and Efficient Simulation of Dynamical Systems, Springer-Verlag, pp. 191-223, 2001. 